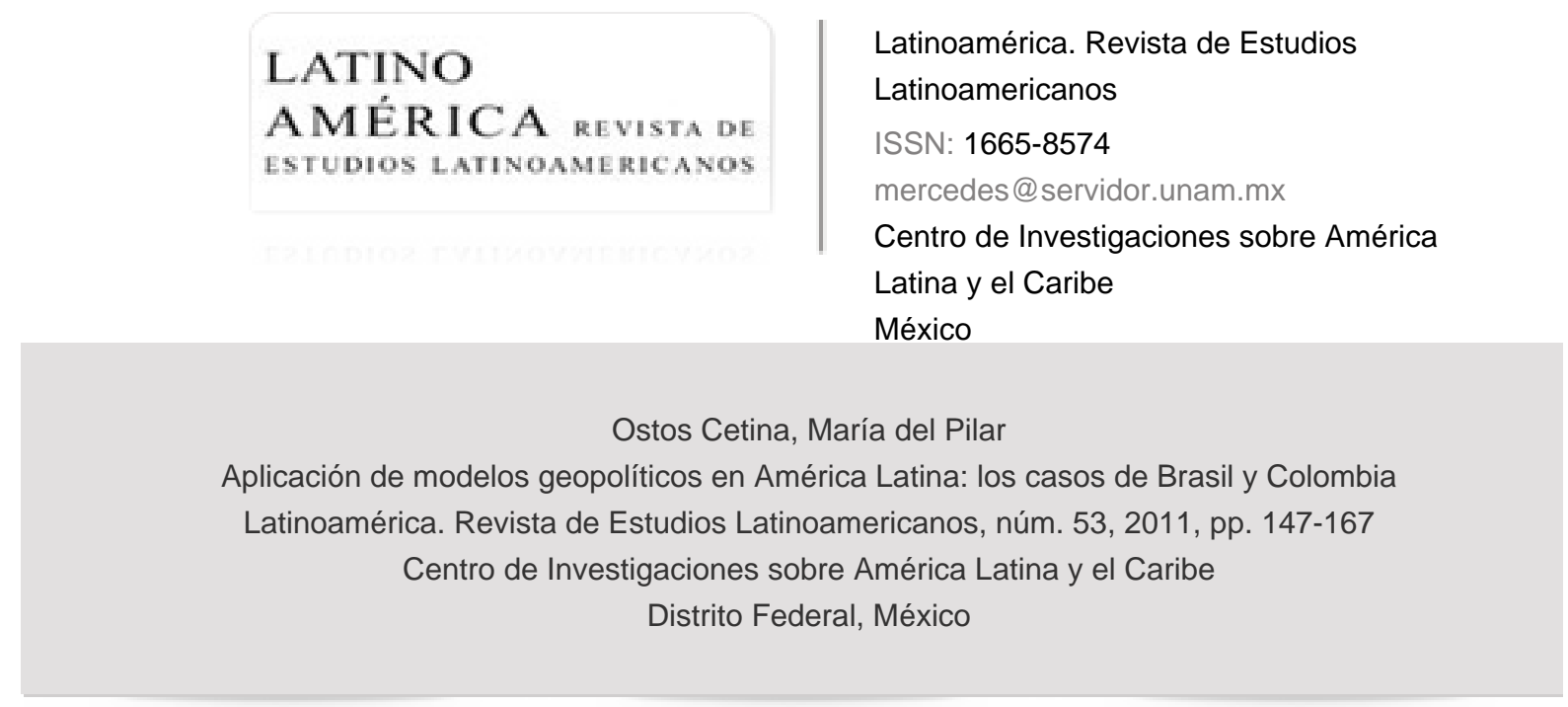

Disponible en: http://www.redalyc.org/articulo.oa?id=64021405008

- Cómo citar el artículo

- Número completo

- Más información del artículo

- Página de la revista en redalyc.org

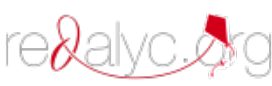

Sistema de Información Científica

Red de Revistas Científicas de América Latina, el Caribe, España y Portugal Proyecto académico sin fines de lucro, desarrollado bajo la iniciativa de acceso abierto 


\title{
Aplicación de modelos geopolíticos en América Latina: los casos de Brasil y Colombia
}

\author{
María del Pilar Ostos Cetina*
}

Resumen: El presente artículo tiene como propósito retomar varios de los elementos fundamentales que brinda la geopolítica clásica con el fin de explicar la nueva reconfiguración de América Latina, a partir de la competencia de intereses hegemónicos que han dado lugar a que dentro de este diseño geopolítico, páises como Colombia, ubicado geográficamente entre el mar Caribe y los territorios que conforman la cuenca andino-amazónica, se haya convertido en una especie de cerco estratégico (rim/and) desde el cual se pretende "vigilar" las acciones de su vecino inmediato: Brasil, a quien se le podría considerar desde esa misma perspectiva el área pivote (heartland) más estratégica de toda la región sudamericana.

PalaBRAS ClavE: Geopolítica clásica, Colombia, Brasil, Intereses hegemónicos, Corazón terrestre, Tierra orilla.

ABSTRACT: This Article has several purposes upon return of the key elements provided by the geopolitics classical, to explain the new re-configuration of Latin America from the competing interests hegemonic, which have led to this design in geopolitics, passing like Colombia, located between the Caribbean Sea and territories that make up the Andean basin Amazonian, have become a kind of fence strategically (rimland) from which it intends to "supervise" the actions of its immediate neighbor: Brazil, who may consider it from that perspective, the pivot area (heartland) more strategically throughout the South American region.

KEy woros: Geopolitics classical, Colombia, Brazil, Interest hegemonic, Heartland, Rimland.

Becaria posdoctoral de la Coordinación de Humanidades (mpostos@yahoo.com). 


\section{INTRODUCCIÓN}

$\mathrm{E}$

1 viraje que tuvo la geopolítica, luego de resistir la estigmatización por sus aparentes vínculos con la propaganda nazi en los primeros decenios del siglo xx, trajo a la par de los estudios en Relaciones Internacionales, nuevos métodos de análisis sobre las explicaciones que desde entonces han merecido los asuntos que versan sobre los conflictos, la guerra y la paz, entre los estados del orbe mundial.

En lo que se refiere al caso en específico de los estudios geopolíticos, sus antecedentes nos remiten a los aportes que hicieron en el campo de la filosofía política pensadores de la talla de Hobbes, Maquiavelo, Montesquieu, Hegel, Kant, entre otros estudiosos de lo político, quienes se dieron a la importante tarea de relacionar factores clave para la geopolítica como son: el territorio, el poder y la sociedad.

En ese mismo sentido, las contribuciones de la geografía aplicada hechas por los científicos alemanes, Alejandro von Humboldt y Karl Ritter, alentaron la visión organicista del Estado, según la cual, el comportamiento de éste se asemejaría a las funciones que sigue cualquier ser vivo, propuesta que contribuyó ampliamente al arranque formal de lo que se conocerá en adelante como: la geopolítica clásica.

De este modo, y al considerar que los estudios geopolíticos emanan de un pensamiento eurocéntrico y anglosajón, liderado por las principales potencias que se disputaban el control y el reparto de zonas de influencia en el ámbito mundial, antes y después de iniciado el siglo xx, el presente ensayo tiene como finalidad retomar varias de las aportaciones que hicieron sus máximos exponentes. Todo esto con el propósito de explicar los acontecimientos que han dado lugar a que en los últimos tiempos en América Latina, países como Colombia, ubicado geográficamente entre el mar Caribe y los territorios que conforman la cuenca andino-amazónica, se hayan convertido en una especie de cerco estratégico (rimland) desde el cual se pretende "vigilar" las acciones del país vecino, en este caso de Brasil, a quien se le podría considerar desde esa misma perspectiva como el área pivote (heartland) más estratégica de toda la región sudamericana 


\section{HaCia la APliCaCión de modelos geopolíticos en América Latina}

Para aquellos que se convirtieron en los pioneros de la llamada geopolítica clásica como fue el caso del sueco Rudolf Kjellen (1864-1922), quien se encargó de proponer a la geopolítica como parte de las ciencias políticas o de las ciencias del Estado en transposición a la rama de la geografía política, su principal propósito fue el de proponer un método que fuera más allá de lo descriptivo, y que en adelante ofreciera un mayor rigor analítico en torno a la relación entre el Estado y el territorio. ${ }^{1}$

Ante este inminente distanciamiento metodológico que se buscaba establecer entre la geografía y la geopolítica, ciertamente persistió la visión organicista del Estado, misma que el propio Kjellen retomó al considerar "al Estado como un ser vivo, cuyo gobierno es el alma y el cerebro, el imperio es el cuerpo y el pueblo son los miembros", el cual cumple con las funciones de nacer, crecer y morir en medio de luchas y conflictos biológicos (raza). ${ }^{2}$

En esa misma tesitura, el alemán Federico Ratzel ${ }^{3}$ (1844-1904) incluyó otra variable clave dentro del análisis geopolíico como es la historia, ya que ésta se encarga de vincular al tiempo y a los hechos del pasado con los acontecimientos presentes y futuros, a partir de los cuales se comprende con mayor veracidad al Estado dentro de una lógica racional y humana, que obliga a pensarlo como ya se mencionó antes, bajo la idea de ser éste un "organismo vivo" que cumple con todas las funciones biológicas de la vida. ${ }^{4}$

1 Carlos de Meira Mattos, Teorías de fronteras, Buenos Aires, Círculo Militar, 1997, p. 26.

2 Se le atribuye a Kjellen la autoría del término Geopolitik (1898), empleado desde su perspectiva para instituir una ciencia empírica del Estado. Rudolf Kjellen, "Autarquía", en Augusto Rattenbach, Antología geopolítica, Buenos Aires, Pleamar, 1975, p. 55.

3 Para Ratzel, la geopolítica es el producto de la interacción dinámica de tres factores: políica, geografía e historia, en Carlos de Meira Mattos, Geopolítica e modernidade. Geopolítica brasileira, Río de Janeiro, Biblioteca do Exército Editora, 2002, p. 18.

4 Meira, op. cit., p. 43; Federico Ratzel, "Ubicación y espacio", en Rattenbach, op. cit., p. 33; Joan Nogué Font y Joan Vicente Rufí, Geopolítica, identidad y globalización, Barcelona, Ariel Geografía, 2001, pp. 34 y 35. 
María del Pilar Ostos Cetina

En ese mismo sentido, Ratzel afirmó que:

La historia nos permite conocer la transitoriedad de los grandes pueblos [... ]. Por ello vemos desaparecer con mayor velocidad a aquellos grupos humanos a quienes la naturaleza misma les niega posibilidades de expansión: pueblos insulares o aquellos que se conformaron con pequeños territorios o, finalmente, agrupaciones humanas reducidas que abarcan amplios espacios sin explotarlos totalmente. ${ }^{5}$

Con esa idea, la historia de Colombia sirve para comprobar el escaso interés de su dirigencia por conservar los territorios legados del periodo precolombino, durante el cual sus primeros habitantes, los chibchas, se extendieron hasta los actuales territorios de Costa Rica y Nicaragua, al grado de que en la actualidad su extensión abarca un espacio mucho menor, esencialmente el corredor andino y la parte de la región Caribe, esto incide en que se sigan considerando como "espacios vacíos" las amplias regiones de los llanos de la Orinoquía y los que comprenden la región amazónica.

En contraste, el relato histórico de Brasil desde la Colonia hasta los días actuales da cuenta de la importancia que reviste para su dirigencia el ocupar aquellos territorios "vacíos" del interior del país. Los cuales comenzaron a ser colonizados por los llamados bandeirantes (aventureros), quienes durante años se encargaron de ocupar las desoladas tierras haciendo uso del principio del $u t i$ possedetis, según el cual, la tierra es de quien la ocupa primero.

Al retomar estos mismos elementos que demuestran la importancia de la ubicación geográfica en el comportamiento del Estado podemos introducir el concepto de "Estado tapón o amortiguador", que acompaña la condición que adquiere, por ejemplo, Colombia con relación a los demás países latinoamericanos desde sus primeras etapas de formación en la época precolombina.

En ese sentido, y siguiendo al geopolítico brasileño, Carlos de Meira Mattos, se trata de un concepto concebido entre ciertos estados que, gracias a su ubicación geográfica, tienden a ser creados para evitar conflictos, mediar y separar

5 Ibid., pp. 44 y 45. 
actores antagónicos, condición misma que lo convierte en un espacio neutro, que adquiere el estatus de Estado. ${ }^{6}$

Obviamente que el devenir histórico y político de Colombia no es justamente producto de este tipo de experiencia, en el que su formación deviene de las disputas entre más estados contiguos, sin embargo, la utilidad operativa de este concepto encuentra algo más que un punto de coincidencia. Si observamos que las características físicas de su territorio, por un lado, el corredor montañoso de los Andes que divide al país longitudinalmente, y por el otro, la muralla de selvas que lo rodean en el extremo noroccidental del lado de la frontera con Panamá y al sur con la selva amazónica en los límites con Perú y Brasil, podemos decir que le otorgan, desde el punto de vista interno, pero también continental, las condiciones necesarias para que el espacio que ocupa lo convierta en un "Estado tapón".

La ubicación de Colombia amurallada por selvas, dividida por la Cordillera de los Andes, inmersa en un conjunto de ríos internos muchas de las veces inexplorados e inutilizables para la navegación fluvial, es fundamental en la conformación de las distintas regiones que coexisten y configuran al país en el que se localizan "islas humanas" diferenciadas entre sí, y que el propio geopolíico colombiano, Julio Londoño ha bautizado con el nombre de "archipiélagos biológicos". 7

Lo anterior se comprueba también a través de la historia, precisamente a partir de la formación del llamado Imperio chibcha, establecido en los actuales territorios de Panamá, Colombia y Venezuela, convertido en una especie de obstáculo geográfico que limitó los acercamientos y las interacciones entre los otros dos imperios más relevantes de la época: el azteca y el inca. Hoy en día, este

6 Así, dotado de soberanía propia, el Estado-tapón, reconocido por los oponentes y en la mayoría de los casos por la comunidad internacional, abriga a un pueblo autónomo y es verdaderamente una unidad independiente. Existen ejemplos de este tipo de estados que fueron constituidos para neutralizar diferencias eminentes y conflictos, tal como fue el caso de Uruguay, que se estableció a propuesta británica de común acuerdo en 1830 entre Brasil y Argentina tras un largo periodo de desavenencias. Ibid., pp. 62 y 63.

7 Véase más sobre el concepto de archipiélago biológico en Julio Londoño, Nueva geopolítica de Colombia, Bogotá, Publicaciones de las Fuerzas Militares, 1948, p. 109. 
mismo antecedente refuerza las pretensiones estadounidenses que convierten a Colombia en su rimland, por ser éste un punto intermedio entre el conglomerado de países que convergen en Centroamérica y el mar Caribe y aquellos otros ubicados del lado sudamericano.

En ese mismo sentido, la dimensión que adquiere el espacio de acuerdo con Ratzel, caracteriza el vínculo existente entre el suelo y el hombre, que al pasar por el espectro de lo político-militar nos conduce a identificar lo que él mismo denominó: "el espacio vital" (lebensraum), que no es más que aquella característica atribuida particularmente a los pueblos más dinámicos y activos con aspiraciones expansionistas, hegemónicas y de conquista, que pretenden alcanzar el beneficio y el dominio de espacios ajenos ocupados por otros pueblos, ${ }^{8}$ tal como se ilustra en los casos de Estados Unidos, Brasil, la ex Unión Soviética, la Alemania Nazi del III Reich, y de otros casos más a lo largo de la historia universal.

Tal característica no podría ser atribuida explícitamente al caso de Colombia, sin embargo, sí conserva un cierto vínculo con lo que Ratzel argumentó sobre aquellos pueblos que, dado el espacio que poseen y su proximidad respecto a otros, hacen que se presenten relaciones más variadas, dinámicas e incluso conflictivas, tal como se observa entre las cinco fronteras terrestres que comparte Colombia con los países vecinos; como lo demuestran los efectos negativos que trajo consigo el desarrollo mismo de un conflicto armado que se inició en dicho país a partir de la segunda mitad del siglo xx.

Otros planteamientos que tienen vigencia son los que expuso el geopolíico Alfred Thayer Mahan (1840-1914), ${ }^{9}$ cuyo legado en esta materia se sintetizó en el diseño de una estrategia de poder, fincada en el poderío naval y en el uso de tácticas militares como base de la proyección internacional de aquellos estados destinados a cumplir con un papel relevante en el ámbito mundial.

Así, el pensamiento geopolítico de Mahan y el diseño táctico que elaboró fue el resultado de sus acuciosos estudios históricos sobre los aciertos que en el

8 Ibid., pp. 15 y 44.

9 Arturo Ponce Urquiza, "El concepto de geoideología desde el pensamiento del evangelista del poder marítimo, Alfred Thayer Mahan", en Rosario Rodríguez Díaz [coord.], El Caribe entre México y Estados Unidos, México, umsnh-Instituto Mora, 2005, pp. 377-390. 
pasado tuvieron tanto la marina inglesa como la marina imperial española. Sobre esta última, Mahan rescató del que fuera "el modelo en forma de cruz" implementado por los españoles en el Nuevo Mundo, varias de las estrategias a seguir por parte de la dirigencia política estadounidense de finales del siglo xIx y principios del xx, en lo que se refiere al establecimiento de puestos de avanzada (bases militares), y el control de las principales rutas destinadas al comercio mundial.

Inicialmente el modelo en cruz se trazó de forma horizontal para establecer la conexión directa entre las Filipinas con las islas Canarias, es decir, entre Asia y Europa, pasando por el punto cardinal de esta ruta, en la que fuera la Nueva España (México), dando lugar también a la correlación de poder de manera vertical entre los territorios norteamericanos y los que convergen en la parte sur hasta alcanzar Tierra del Fuego. La operatividad de este modelo en cruz a comienzos del siglo xx, a sugerencia del propio Mahan, llevó a los estadounidenses a procurar la salida de la presencia española de sus ex colonias, tanto del lado asiático en las Filipinas como de las islas de Cuba y Puerto Rico en el mar Caribe. Una vez habilitado este corredor entre Asia-Pacífico y Europa pasando por el Caribe, el siguiente paso consistió en ubicar el punto cardinal que se emplearía para coordinar las operaciones del área siendo en este caso, ya no la Nueva España sino Panamá, luego de alcanzar su independencia del territorio colombiano en 1903, conformando así el lugar idóneo desde el cual los estadounidenses se proponen "abrazar el mundo", hecho que se reafirma tras la construcción del Canal pocos años después.

Desde entonces, Panamá se erigió como ese gran epicentro de poder estadounidense en el continente, desde donde se coordinarían las labores de inteligencia, aprovisionamiento y entrenamiento militar en la región, además de convertirse en la zona de tránsito obligada para el comercio internacional; lo cual en términos geopolíticos, no es otra cosa que el ejemplo más claro de lo que se conoce como la formación y establecimiento de un verdadero rimland (o tierra orilla), tal como lo expone más adelante el teórico judío-holandés Nicholas Spykman

Cabe destacar que la formulación de un rimland por parte de Spykman va a ser la respuesta a lo planteado previamente por el británico Halford Mackinder 
(1861-1947), quien expuso acerca del valor geoestratégico de lo que consideró la formación de una "gran isla territorial" con el nombre de "Eurasia", producto de la fusión entre dos continentes: Europa y Asia. Lo que a su vez se convierte en una especie de beartland (el corazón de la tierra), dentro del cual prevalece un centro o pivote, que para el caso citado de Eurasia se asocia con el valor estratégico que adquiere un lugar como Rusia.

Desde esta lógica, en su condición de inglés, Mackinder se proponía el establecimiento de una alianza entre las potencias del mar: Reino Unido, Canadá, Estados Unidos, Sudáfrica, Australia y Japón, además de un acercamiento y la firma de alianzas con los países de Europa del Este para cercar y debilitar el área pivote (Rusia); situación que terminó por incidir en los hechos que motivaron el inicio de la Primera y Segunda guerras mundiales, lo mismo que la Revolución rusa en los primeros decenios del siglo $\mathrm{xx}^{10}$

Frente a la propuesta inglesa a través del pensamiento geopolítico de Mackinder, aparece una contrarrespuesta desde Estados Unidos por intermedio de Nicholas J. Spykman (1893-1943), quien al poner en duda el núcleo duro de la Teoría del Corazón de la Tierra, se encargó de asentar los lineamientos de lo que se conocerá como: la Teoría del Rimland (la teoría del cerco o la tierra-orilla), que consiste en determinar un área-tapón que funcione como una amplia zona amortiguadora en el conflicto entre el poder marítimo y el poder terrestre.

Así, esta propuesta teórica llevada a la praxis se convertiría en una de las principales estrategias implementadas por Estados Unidos tras la posguerra, al afirmarse que "quien controla el rimland domina Eurasia, y quien domina Eurasia controla los destinos del mundo". ${ }^{11}$ La validez de este precepto teórico, según Spykman, se sustenta en el valor estratégico que apreció en todos aquellos estados o espacios que, de acuerdo con su situación geográfica, se localizan en mayor o menor proximidad al área pivote, a los océanos, masas continentales, a la posición que ocupan respecto a la línea del Ecuador, a los centros de poder, a las

${ }^{10}$ Antonio Cavalla, La geopolítica y el fascismo dependiente, Santiago de Chile, Casa de Chile, 1977, p. 25.

${ }^{11}$ Leonel Itaussu Almeida Mello, Quem tem medo da geopolítica?, São Paulo, Hucitec, 1999, pp. 119-126. 
áreas de conflicto, a las grandes rutas de comunicación y a su emplazamiento con relación a sus vecinos inmediatos. ${ }^{12}$

Con lo cual, la concepción de Spykman se fundamenta en términos de lo que representa la ubicación geográfica de cualquier Estado, así este factor resulta uno de los más analizados por la escuela geopolítica alemana con Ratzel y otros, quienes van a influir sustancialmente al momento de determinar aquellos puntos geoestratégicos desde los cuales se puede "cercar y controlar" al área pivote.

De esta manera, al intentar relacionar ambos modelos, tanto el de Mackinder como el de Spykman, aplicados al contexto actual latinoamericano, se logran vislumbrar los siguientes aspectos. Por un lado, las condiciones geoestratégicas que convierten actualmente a Brasil en una especie de "Corazón de la tierra" al interior de todo el macizo continental sudamericano, dentro del cual prevalece una región-pivote, tal como podría ser considerada la llamada cuenca andino-amazónica. ${ }^{13}$ Frente a esta realidad, las circunstancias geográficas, histórico-políticas y de la hegemonía regional encabezada por Estados Unidos, le otorgan a Colombia la condición de "cerco estratégico" (rimland) o de línea de defensa para llevar a cabo diferentes actividades y maniobras como parte de su inminente vecindad con Brasil, considerado desde esta perspectiva de análisis en el actual beartland sudamericano.

LAS APORTACIONES DE LA ESCUELA GEOPOLÍTICA BRASILEÑA

A LA COMPRENSIÓN DE BRASIL COMO UN HEARTLAND SUDAMERICANO

Tras abordar varios de los fundamentos teóricos que propone la geopolítica clásica, y situar a Brasil como una especie de heartland y a Colombia como rimland, a continuación se propone considerar algunas de las opiniones que sobre

${ }^{12}$ Laura Muñoz, Geopolítica, seguridad nacional y política exterior. México y el Caribe en el siglo XIX, México, IIH/UMSNH, 2001, pp. 29 y 30.

${ }^{13}$ En ésta convergen territorios de ocho países: Bolivia, Brasil, Colombia, Ecuador, Guyana, Perú, Surinam y Venezuela, que comprende un área total equivalente a las 2/5 partes del territorio sudamericano y poseen la quinta parte de disponibilidad de agua dulce del mundo, lo que permite que se produzca más de 40\% del oxígeno mundial. En Carlos de Meira Mattos, Uma geopolítica pan-amazónica, Río de Janeiro, Livraria José Olympio Editora, 1980, p. 120. 
el mismo tema plantearon quienes integraron la llamada "Escola Geopolítica Brasileira".

De este modo, uno de los pioneros de esta escuela del pensamiento geopolítico fue la del general brasileño Mario Travassos, quien durante la década de los treinta del siglo xx, en su trabajo "La proyección continental del Brasil" ${ }^{14}$ afirmó que uno de los principales factores a vencer por parte de la dirigencia política de Brasil, para encaminar su destino como una nación líder en la región, requería vencer dos obstáculos: el primero, llevar a cabo una política de poblamiento de todos aquellos espacios "vacíos" al interior del extenso territorio, acompañado de una adecuada infraestructura vial y de comunicaciones. ${ }^{15} \mathrm{Y}$ en segundo lugar, identificar sus salidas al mar, superando la condición antagónica del Atlántico y del Pacífico separados por la cadena montañosa de los Andes y en otro sentido, superar el "antagonismo vertical", entre la región amazónica y la cuenca de la Plata, creando un tercer espacio para balancear su poder continental a través de su influencia en la zona boliviana de Cochabamba y Santa Cruz de la Sierra; de esta manera conformar un triángulo estratégico desde el cual se proyectara continentalmente. ${ }^{16}$

Desde esta misma visión, otro de los miembros de esta escuela fue el también general Carlos de Meira Mattos, ${ }^{17}$ quien afirmó que "el destino del Brasil está

${ }^{14}$ Fue general de la división del Ejército brasileño en la década de los treinta del siglo xx. Sus principales obras fueron: Mario Travassos, Projeçao continental do Brasil, São Paulo, Compañía Editora Nacional, 1935

${ }_{15}$ Para Travassos, la manera de superar los obstáculos que presenta la geografía sudamericana guardaban una estrecha vinculación con la creación de una política de transporte (red ferroviaria, capaz de conectar los puertos con el interior del territorio) que neutralice, a favor del Brasil, los desequilibrios ofrecidos por los antagonismos fisiográficos existentes, en Meira, op. cit., p. 60.

${ }^{16}$ Jorge Mantel Da Costa Freitas, A escola geopolítica brasileira, Río de Janeiro, Biblioteca do Exército Editora, 2004, pp. 17-19.

${ }^{17}$ Nació en San Carlos, Estado de São Paulo, Brasil, el 23 de julio de 1913. Se integró desde muy joven a la Escuela Militar, formó parte de la Fuerza de Expedición Brasileña (FEB) que operó en Italia. A su regreso, continuó una carrera militar en ascenso que lo llevó a ocupar, en 1973, el cargo de general de división y, posteriormente, numerosos puestos de relevancia política y diplomática en su país como en otras naciones. Véanse más detalles biográficos en ibid., pp. 63 y 64 . 
en completa relación con su carácter de país continental, en la que debe velar por su seguridad y el fortalecimiento de sus alianzas continentales y extra continentales, procurando la paz en el continente siendo ésta una de sus prioridades en materia de política exterior". ${ }^{18}$ Por las mismas razones, Meira consideró prioritario integrar al territorio nacional brasileño la región de la Amazonía, ya que para este geopolítico es, precisamente, ahí donde se inicia la "continentalización de la binterlandia sudamericana", basada en la utilización de la tecnología de los transportes y de las comunicaciones encaminada al progreso y al desarrollo económico de América del Sur. ${ }^{19}$

Ciertamente, este legado geopolítico encuentra sustento en varias de las apreciaciones que tiempo atrás fueron formuladas por personajes de la vida política, diplomática y militar de Brasil, comenzando por el propio José Bonifacio, quien fuera el jefe de gobierno durante el reinado de Pedro I, cuya plataforma geopolítica para Brasil consideró, entre los aspectos más apremiantes de su administración, la de fundar una ciudad al interior del amplio territorio brasileño para convertirse en sede del gobierno, establecer una política de poblamiento en aquellos espacios vacíos del reino y promover el desarrollo de la actividad minera.

Más tarde, quien ha sido considerado hasta la actualidad el "padre de la diplomacia brasileña", José María da Silva Paranhos (1845-1912), conocido como el Barón de Río Branco, expresó en términos geopolíticos que para alcanzar una proyección continental de la políica exterior de Brasil era necesario considerar a América del Sur como su zona natural y de influencia regional. Lo que a su vez exigía la formulación de un tipo de diplomacia más dinámica y pro activa, fincada en el acercamiento hacia los principales centros de poder mundial y amparada

${ }^{18}$ Véase más en Carlos de Meira Mattos, Brasil, geopolítica e destino, Río de Janeiro, Livraria José Olympio Editora, 1979, p. XV y 147.

${ }^{19}$ Al respecto, Meira ha publicado numerosas obras versadas en la importancia geopolítica del territorio de Brasil en Sudamérica y el continente, a partir de las cuales hace un importante estudio del poder nacional, sus orígenes históricos, su naturaleza y sus manifestaciones geopolíticas; mismas que inciden en la revalorización de la cuenca hídrica del Amazonas como punto estratégico "multinacional", que avizora un acelerado desarrollo socioeconómico en el ámbito regional, en Meira, Brasil, geopolítica..., pp. 79 y 80. 
en un verdadero poder militar que garantice la defensa y la soberanía del amplio territorio del Brasil.

En esa misma tesitura, la geopolítica brasileña, Teresina de Castro ${ }^{20}$ se encargó, desde el campo académico, de establecer las coordenadas geoestratégicas a partir de las cuales se propone para Brasil una mayor atención en dos regiones clave en todo el hemisferio: la región amazónica y la región antártica; lo que implica para la dirigencia política brasileña centrar su atención en tres aspectos: el terrestre, el marítimo y el aéreo. ${ }^{21}$

En función de estos mismos planteamientos, particularmente los que se vinculan con el potencial estratégico de la región amazónica, la geógrafa brasileña Bertha Becker nombró a "la Amazonía como el heartland (corazón) ecológico del planeta", debido a la dimensión de la masa terrestre y de bosques tropicales en el Amazonas - que históricamente han dificultado su ocupacióny que hoy en día lo convierten en un espacio de "autodefensa", que envuelve a la Amazonía ya no sólo de Brasil sino de toda Sudamérica; convirtiéndola en una posición geoestratégica para distintos actores, incluyendo a los científicos, las transnacionales, las farmacéuticas, pero además a las autoridades militares que resguardan esta importante zona donde confluyen las fronteras de los países vecinos y en las que se destaca, por cierto, la presencia de actores armados que participan en el conflicto armado colombiano; un tema que en el último decenio ha adquirido mayor relevancia a partir de la puesta en marcha del llamado Plan Colombia, mismo que incluye la colocación de bases militares con

${ }^{20}$ Nació en la ciudad de Río de Janeiro, el 22 de diciembre de 1930. Obtuvo el grado de licenciada en Geografía e Historia por la Facultad Nacional de Filosofía de la Universidad del Brasil (actualmente la Universidad Federal de Río de Janeiro). Fungió durante cerca de treinta años como docente del Colegio Pedro II, al mismo tiempo colaboró de forma permanente con la Escuela Superior de Guerra, en calidad de adjunta de la División de Asuntos Internacionales. Para ver más datos biográficos de la autora, se recomienda el trabajo de Da Costa Freitas, $o p$. cit., pp. 87 y 88 y el libro de la autora, Teresina de Castro, Nossa América, geopolítica comparada, Río de Janeiro, Biblioteca del Exército Editora, 1994.

${ }^{21}$ Según la autora son tres: "La isla subdesarrollada" (formada por las regiones del norte y el centro este), la segunda llamada: "la isla en desarrollo" (integrada por la zona del noreste del país) y la tercera, "la isla desarrollada" (que incluye la región sur y sureste). Ibid., pp. 90 y 91. 
apoyo estadounidense en los límites fronterizos de Brasil, como en otros países vecinos a Colombia.22

De esta manera, la región amazónica se convierte hasta la actualidad en un espacio estratégico y expectante, ya no sólo para los países vecinos que confluyen en esta cuenca que hace parte de ese heartland brasileño sino que, además, tiende a revestir un notable interés en los planes hegemónicos de Estados Unidos, al grado de convertir a Colombia en esa especie de rimland o de puesto de avanzada desde el cual se propone "vigilar" y atender todo aquello que se presente en la que consideran hasta el día de hoy, su principal zona de influencia en todo el mundo.

LA INFLUENCIA DE LOS PLANES HEGEMÓNICOS DE ESTADOS UNIDOS

EN EL DEVENIR GEOPOLÍTICO DE COLOMBIA Y BRASIL

Al considerar ciertos aspectos de la propuesta de Mahan, la dirigencia estadounidense se ha encargado de establecer un amplio conjunto de puntos de avanzada, convertidos en centro de operación y coordinación de actividades para la defensa del continente, algo que se inició en Panamá, y hoy en día Florida es sede del Comando del Sur y se extiende hacia otros puntos de la geografía latinoamericana comenzando por la base radar de Guantánamo (Cuba), seguida de las bases de Liberia en Costa Rica, Sotocano en Honduras, Vieques en Puerto Rico, Comapala en El Salvador, además de la base radar en Aruba e Isla Margarita. Esta misma presencia militar estadounidense se extiende en Sudamérica hasta Surinam, pasando por las bases militares en Colombia que, inicialmente, se establecieron del lado de los llanos y la Amazonía en sitios como Caquetá, Larandida y Tres Esquinas (a las cuales se sumaron otras siete bases militares a partir de 2008, tras la negativa de Ecuador de firmar el contrato de prórroga de la base mi-

22 Bertha Becker, Amazonía. Geopolítica na virada do III milenio, Río de Janeiro, Garamond Ltda., 2004, p. 33 y en Diana Marcela Rojas y Atehortua Adolfo León, "El Proceso de paz y el Plan Colombia", en El Plan Colombia y la internacionalización del conflicto, Bogotá, Instituto de Estudios Políticos y Relaciones Internacionales (IEPRI), Universidad Nacional de Colombia, Planeta, 2001. 
litar de Manta a Estados Unidos). ${ }^{23}$ En Perú se encuentran las bases militares de Iquitos y Santa Lucía, mientras que en Bolivia se mantiene en funcionamiento la unidad antiterrorista en Santa Cruz de la Sierra. Estas mismas se conectan con el área de la Triple Frontera en la que confluyen los países de Paraguay, Argentina y Brasil, siendo Chubut (Argentina), un importante centro para las labores del personal estadounidense que dirige el sistema de espionaje satelital "Echelón", aunado a la presencia que tienen en Tierra del Fuego, punto de avanzada de cara a la región antártica.

El resultado de este enorme cinturón de seguridad, extendido por los estadounidenses en toda América Latina y el Caribe, refuerza de forma significativa sus intereses y pretensiones sobre la vasta región del Amazonas, donde Brasil se convierte en el poseedor de cerca de $67 \%$ del total de esta región, considerada por muchos expertos como el principal pulmón del mundo y la más importante fuente de agua dulce y de diversidad del planeta. A lo cual habría que atribuir otros méritos, respecto a su capacidad de producción de recursos estratégicos, entre los que se destacan los recientes hallazgos de petróleo, localizados en aguas profundas en la zona conocida como el Pre-sal, frente a las costas de São Paulo y Río de Janeiro. Todo esto sin dejar de mencionar el potencial brasileño en la producción de biocombustibles, derivados del procesamiento de la soja y la caña de azúcar, lo cual junto con la producción de café y carnes, convierten a Brasil entre las primeras economías del mundo.

Estas coyunturas alientan, sin duda alguna, el papel de Colombia al interior de los planes de control previstos por los norteamericanos, en el cual se sitúa a Brasil como ese heartland sudamericano. Situación que no excluye la posibilidad de atender, desde la propia Colombia, otros puntos de interés de los estadounidenses. Expresión de ello es la propia Venezuela, cuya importancia radica en su creciente poderío petrolero concentrado en la que se conoce como la faja del Orinoco. Hecho que a su vez explica, la manera de actuar de la administra-

${ }^{23}$ De este modo, a partir de 2009 se propuso el establecimiento y adecuación de las bases de Malambo, en el extremo norte cerca de la costa atlántica y con dominio sobre el mar Caribe, la de Apiay, en el centro-sur, que controla la Orinoquía y la Amazonía, Palanquero y Tolemaida, convertidas en instalaciones aéreas, en la zona central de este país andino. 
ción de gobierno de este país, encabezada por el mandatario Hugo Chávez, quien proyecta los intereses de gobierno a través de un connotado activismo políico y diplomático, tendiente a retomar del pasado los planes de integración bolivariana en todo el conjunto de países latinoamericanos y caribeños, como una manera de romper con el equilibrio de poder y orden dictados por Washington. ${ }^{24}$

Lo anterior explica por qué el actual mandatario venezolano ha dispuesto, a lo largo de sus poco más de diez años de gestión, desarrollar un vasto plan de modernización de sus fuerzas armadas, siendo ésta una forma de contrarrestar la presencia estadounidense en el territorio colombiano, y a través de la búsqueda de nuevos aliado-proveedores para la compra de material bélico como se ha hecho con Rusia, país al que Venezuela le ha comprado 24 aviones de combate Su-30 y 53 helicópteros militares por un monto total de 3000 millones de dólares. Esta compra se produjo luego de que Estados Unidos prohibiera la venta de armas a Venezuela (cuando tradicionalmente era su principal proveedor de equipamiento militar) e impidiera que España le vendiera aeronaves militares con tecnología estadounidense, a pesar de lo cual España venderá a Venezuela cuatro barcos de patrullaje. Independientemente de las objeciones de Estados Unidos, Rusia vendió al ejército venezolano, 10000 fusiles AK-47 y está construyendo una fábrica de municiones kalashnikov en el país. Cabe destacar que a pesar de estos hechos, el suministro de petróleo de Venezuela hacia su principal comprador de petróleo, en este caso Estados Unidos, no se ha visto alterado de modo alguno..$^{25}$

Respecto a Brasil y su relación con Estados Unidos, durante la administración reciente de Luis Ignacio Lula, se puso en marcha un plan que conjunta los esfuerzos de su gobierno con el de importantes empresarios brasileños que se dedican a la producción a gran escala de soja y caña de azúcar, productos a partir de los cuales se obtiene como resultado final el etanol (biodisel); mismo que

${ }^{24}$ Valentina Lares Martiz, "Una mirada al ajedrez petro-político del presidente de Venezuela, Hugo Chávez", en el periódico El Tiempo, Bogotá, 2006.

${ }^{25}$ Al respecto véase, Socorro Ramírez, "Colombia y sus vecinos", en Nueva Sociedad, núm. 162, Caracas, julio-agosto, 2004. 
se empieza a comercializar con notable éxito en aquellos mercados de alta demanda de combustibles, tal como se presenta en el caso estadounidense. ${ }^{26}$

Por todo esto es de suponer que la pretensión norteamericana para los tiempos futuros consistirá en demandar energías "limpias, renovables y económicas", que se obtengan de la producción masiva de semillas de girasol, soja, caña de azúcar, maíz, palma africana, cultivadas en Brasil y en otros países aptos para este tipo de cultivos tropicales, que pueden ser comprados a precios mucho más "redituables", frente a los elevados costos que hoy se destinan a la adquisición de petróleo.

En vista de lo anterior, la perspectiva de la dirigencia estadounidense no solamente consistirá en ampliar su "matriz energética", mediante el uso de los biocombustibles, sino que además, en términos de control político en su zona de influencia natural, buscará trascender el impasse ocasionado tiempo atrás por la negativa del propio Brasil y de otros países de la región frente a la creación del Área de Libre Comercio de las Américas (ALCA), al establecer otro tipo de acuerdos y de compromisos, si se quiere bilaterales o trilaterales, tal como se vienen desarrollando en los últimos tiempos a partir de la firma del Tratado de Libre Comercio (TLC) entre Estados Unidos y México, pero también como se viene adelantando con países en la región como son los casos de Costa Rica, Panamá, Colombia, Chile, entre otros.

Aunado a este tipo de mecanismos empleados por Estados Unidos en la diversificación de sus relaciones y en los criterios de producción que asigna a los países en América Latina, se creó también la "Comisión Interamericana del Etanol", ${ }^{27}$ en la que participa Brasil. En este mismo tenor cabe mencionar el con-

${ }^{26}$ En 2006, Brasil exportó 58\% de su producción de caña de azúcar y 85\% de soja al mercado estadounidense. En http://www.ecoportal.net.

27 "El 18 de diciembre de 2006, en el Biltmore Hotel de Miami, el todavía gobernador Jeb Bush, Roberto Rodrigues, presidente del Consejo Superior de Agronegocios de San Pablo y ex ministro de Agricultura del gobierno de Lula, y Alberto Moreno, presidente del Banco Interamericano de Desarrollo (BID), anunciaron la formación de la comisión "que tiene como misión fomentar el uso del etanol en las mezclas del nafta en el continente americano". Véase más sobre el tema en Raúl Zibechi, "Estados Unidos y Brasil: la nueva alianza etanol", en Programa de las Américas del International Relations Center (IRC), 6 de marzo, 2007. 
junto de visitas de Estado que realizó en su momento el entonces presidente, George Bush a Brasil, Uruguay, Colombia, Guatemala y México, en los primeros meses de 2007, con el propósito de asegurar en cada uno de estos países las fuentes reales para la producción, aprovisionamiento y distribución de estas nuevas energías, derivadas de variados cultivos que en esencia hacen parte de la base alimenticia de los pueblos de la región latinoamericana, y que ahora harán parte de esa nueva dinámica productiva que convertirá dichos productos de consumo humano, en recursos codiciables por su valor energético en el ámbito mundial.

Pero mientras Estados Unidos gestiona y asegura a sus principales proveedores de biocombustibles, la dirigencia brasileña y los empresarios dedicados al sector agrario buscan potencializar su política agroindustrial allende sus fronteras, proyectando sus iniciativas en México y algunos países centroamericanos y caribeños (Honduras, Nicaragua, Jamaica y Panamá), los cuales formaron parte del conjunto de visitas de Estado realizadas por el presidente Lula, en compañía de un grupo de empresarios brasileños. El propósito central de cada una de estas visitas fue la de estrechar los vínculos de cooperación entre Brasil y cada uno de estos países en materia de transferencia tecnológica para la explotación del petróleo a través de su estatal Petrobrás, y, en otros casos, se trató de promover asistencia técnica en distintos ámbitos de la producción agrícola como fue el caso de la planta de deshidratación de etanol, inaugurada por el presidente Lula, en Port Esquivel (Jamaica). Mientras que en Panamá, el gobierno de Brasil manifestó un gran interés por invertir y participar, junto con sus empresarios, en las licitaciones para la ampliación de las esclusas que conforman el Canal de Panamá. ${ }^{28}$

Desde esta perspectiva se puede percibir en el futuro cercano que, tanto Brasil como Venezuela pretendan alcanzar un amplio liderazgo cada uno en la producción de energías derivadas de los agrocombustibles y del petróleo respectivamente, un aspecto que, analizado desde un enfoque geopolíico, incide

${ }^{28}$ En Luis Ignacio Lula da Silva, "Brasil-México: una asociación necesaria", en Embajada del Brasil en México, 9 de agosto, 2007. 
en la persistencia de ambos países por superar el obstáculo geográfico de no contar con una salida directa sobre las costas del océano Pacífico, punto de salida que favorecería el envío de sus bienes estratégicos hacia otras partes del mundo, en particular a los mercados asiáticos y los de Medio Oriente, además de Rusia. Otro aspecto a examinar es que frente al reducido interés que actualmente manifiesta la dirigencia mexicana en Centroamérica y en el Caribe, considerada históricamente su tradicional zona de influencia, países como Venezuela y Brasil se disputen su supremacía en esta misma región, aplicando todo tipo de estrategias como se ha venido presentando tras la firma de acuerdos de cooperación técnico-científico, inversiones en materia social e infraestructura, requerida por buena parte de los países que conforman esta subregión.

Otro aspecto que se deriva de esta nueva dinámica productiva en materia de energéticos y de la competencia por el liderazgo regional, se encuentra estrechamente vinculado al tema militar y de seguridad, que en la perspectiva estadounidense se promueve desde la propia posición que adquiere actualmente Colombia como centro estratégico para la ejecución de los planes estadounidenses en el continente.

Así, pues, Colombia se convierte en el rimland desde el cual Estados Unidos asegura su proximidad al que podría considerarse su "beartland ecológico", haciendo alusión a la "cuenca andino-amazónica", dentro de la cual Brasil posee $60 \%$ de la selva amazónica, lo que a su vez representa una impresionante riqueza en términos de especies naturales (algunas aún sin descubrir), pero también a importantes fuentes de agua dulce para el consumo humano, que por si fuera poco se busca darle el estatus de "patrimonio de la humanidad".

En respuesta a este interés siempre manifestado por Estados Unidos desde el inicio de sus primeros viajes de exploración en el continente, a partir de la segunda mitad del siglo xIx, y ante su persistente política hegemónica encaminada al aseguramiento de sus principales fuentes en todo el continente, el militar brasileño, Carlos de Meira Mattos advirtió en sus últimas entrevistas sobre la apremiante necesidad que recae en la dirigencia de Brasil para reforzar militarmente sus fronteras. Lo cual se traduce en una mayor vigilancia sobre la selva amazónica frente: 
a las incursiones armadas de la guerrilla colombiana, que constituyen una amenaza para nuestras fronteras, en tanto que en Paraguay, Estados Unidos está creando una base militar, y Venezuela, no se sabe para qué, acaba de comprar material de guerra ultramoderno con el cual se transformará en la mayor potencia militar de América Latina. ${ }^{29}$

En ese sentido, la propuesta del general Meira Mattos consistiría en "militarizar la frontera" que comprende el amplio territorio de Brasil, para estar en consonancia con lo que tiempo atrás había planteado el Barón de Río Branco, quien propuso que para que una negociación diplomática tuviera éxito era preciso estar apoyado en un poder militar adecuado.

Desde este punto de vista, el manejo de las actuales relaciones entre Brasil y Estados Unidos, lo mismo que frente a Venezuela, más allá de los acercamientos comerciales que hayan podido alcanzar, se encuentran permeados por el componente militar de uno y otro lado, haciendo que dentro de este modelo geopolítico, el Estado colombiano pueda seguir siendo considerado por la dirigencia estadounidense su rimland en el corazón del continente americano.

Recibido: 8 de diciembre, 2010. Aceptado: 25 de agosto, 2011.

\section{BibliograFíA}

Almeida Mello, Leonel Itaussu, Quem tem medo da geopolítica?, Hucitec, São Paulo, 1999.

Beaufré, André, Introducción a la estrategia, Buenos Aires, Sthuart and Cia, 1977.

${ }^{29}$ Entrevista al general Carlos de Meira Mattos, "Se rompe el equilibrio militar en Suramérica", en periódico Folba de São Paulo, São Paulo, 24 de octubre, 2006. 
BeCKer, Bertha, Amazonía. Geopolítica na virada do III milenio, Río de Janeiro, Garamond Ltda., 2004.

Castro Teresina de, Nossa América, geopolítica comparada, Río de Janeiro, Biblioteca del Exército Editora, 1994.

Cavalla, Antonio, La geopolítica y el fascismo dependiente, Santiago de Chile, Casa de Chile, 1977.

Costa FREITAS, Jorge Mantel Da, A escola geopolítica brasileira, Río de Janeiro, Biblioteca do Exército Editora, 2004.

CHAu, Marilena, Brasil. Mito fundador y sociedad autoritaria, São Paulo, Fundación Perseo Abramo, 2004.

GonzÁLEZ, Leopoldo, "La mitología estadounidense, sus consecuencias en la política internacional y la forma de neutralizar a sus críticos", en Revista Relaciones Internacionales, México, FCPS-unaM, mayo-agosto, 2006.

Hervández-Vela SALGADo, Edmundo, Diccionario de Política Internacional, México, Porrúa, 2002.

KJELLEN, Rudolf, "Autarquía", en Augusto Rattenbach, Antología geopolítica, Buenos Aires, Pleamar, 1975.

LARES Martiz, Valentina, "Una mirada al ajedrez petro-político del presidente de Venezuela, Hugo Chávez", en el periódico El Tiempo, Bogotá, 2006.

Londoño, Julio, Nueva geopolítica de Colombia, Bogotá, Publicaciones de las Fuerzas Militares, 1948.

Lula DA SIIvA, Luis Ignacio, "Brasil-México: una asociación necesaria", Embajada del Brasil en México, 9 de agosto, 2007.

Meira MatTos, Carlos de, Teorías de fronteras, Buenos Aires, Círculo Militar, 1997. ,Geopolitica e modernidade. Geopolítica brasileira, Río de Janeiro, Biblioteca do Exército Editora, 2002.

, Uma geopolítica pan-amazónica, Río de Janeiro, Livraria José Olympio Editora, 1980.

José Olympio Editora, 1979.

, Brasil, geopolítica e destino, Río de Janeiro, Livraria 
, "Se rompe el equilibrio militar en Sudamérica", en periódico Folba de São Paulo, 24 de octubre,2006.

MuÑoz, Laura, Geopolítica, seguridad nacional y politica exterior. México y el Caribe en el siglo XIX, México, IIH-UNAM/UMsNH, 2001.

Nogué Font, Joan y Joan Vicente Rufí, Geopolítica, identidad y globalización, Barcelona, Ariel Geografía, 2001.

Ponce Urquiza, Arturo, "El concepto de geoideología desde el pensamiento del evangelista del poder marítimo, Alfred Thayer Mahan", en Rosario Rodríguez Díaz [coord.], El Caribe entre México y Estados Unidos, México, umsnh/Instituto Mora, 2005.

Ramírez, Socorro, "Colombia y sus vecinos", en Nueva Sociedad, núm. 162, Caracas, julio-agosto, 2004.

RojAs, Diana Marcela y Atehortua Adolfo León, "El proceso de paz y el Plan Colombia", en El Plan Colombia y la internacionalización del conflicto, Bogotá, Instituto de Estudios Políticos y Relaciones Internacionales (IEPRI)/ Universidad Nacional de Colombia/Planeta, 2001.

Travassos, Mario, Projeçao continental do Brasil, São Paulo, Compañía Editora Nacional, 1935.

UrRego Ardila, Miguel Ángel, La crisis del Estado Nacional en Colombia. Una perspectiva histórica, Morelia, IIH-UMsNH, 2004.

ZiBECHI, Raúl, "Estados Unidos y Brasil: la nueva alianza etanol", en Programa de las Américas del International Relations Center (IRC), 6 de marzo, 2007.

PÁGINA ELECTRÓNICA

http://www.ecoportal.net 